\title{
COLOR SUPERCONDUCTIVITY
}

\author{
THOMAS SCHÄFER \\ TRIUMF, 4004 Wesbrook Mall \\ Vancouver, BC, Canada, V6T2A3
}

\begin{abstract}
We discuss recent results on color superconductivity in QCD at large chemical potential.
\end{abstract}

\section{Introduction}

The phase structure of matter at non-zero baryon density has recently attracted a great deal of interest. The behavior of hadronic matter in this regime is of interest in connection with the structure of compact astrophysical objects and the physics of heavy ion collisions in the regime of maximum baryon density. In addition to that, we are addressing a fundamental question about QCD, namely, what is the ultimate fate of hadronic matter as we keep compressing it to higher and higher densities. And finally, understanding the structure of matter at finite density is part of our quest to understand the full richness of the QCD phase diagram as we vary the temperature, the density, and the number of quark flavors and their masses.

At very high density the natural degrees of freedom are quasiparticles and holes in the vicinity of the Fermi surface. Since the Fermi momentum is large, asymptotic freedom implies that the interaction between quasiparticles is weak. In QCD, because of the presence of unscreened long range gauge forces, this is not quite true. Nevertheless, we believe that this fact does not essentially modify the argument. We know from the theory of superconductivity that the Fermi surface is unstable in the presence of even an arbitrarily weak attractive interaction. At very large density, the attraction is provided by one-gluon exchange between quarks in a color anti-symmetric $\overline{3}$ state $\mathrm{QCD}$ at high density is therefore expected to behave as a color superconductor 1 .

Color superconductivity is described by an order parameter of the general form

$$
\phi=\left\langle\psi^{T} C \Gamma_{D} \lambda_{C} \tau_{F} \psi\right\rangle .
$$

Here, $C$ is the charge conjugation matrix, and $\Gamma_{D}, \lambda_{C}, \tau_{F}$ are Dirac, color, and flavor matrices. Except in the case of only two colors, the order parameter cannot be a color singlet. Color superconductivity is therefore characterized by the breakdown of color gauge invariance. As usual, this statement has to be interpreted with care. Local gauge invariance cannot really be broken 1 . Nevertheless, we can fix the gauge and study, in weak coupling perturbation theory, the implications of a nonzero vacuum expectation value of the type (11). The most important gauge invariant consequence of color superconductivity is the appearance of a mass gap, through the Anderson-Higgs phenomenon. The formation of a mass gap is of course also characteristic of a confined phase. Indeed, if is known that in general Higgs and confined phases are continuously connected $\mathbf{E}$.

In addition to that, color superconductivity can lead to the breakdown of global

mbx: submitted to World Scientific on June 25, 2018 
symmetries. We shall see that in some cases there is a gauge invariant order parameter for the breaking of the $U(1)$ of baryon number. This corresponds to true superfluidity and the appearance of a massless phonon. We shall also find that for $N_{f}>2$ color superconductivity leads to chiral symmetry breaking.

\section{The renormalization group}

In order to assess the relative importance of different instabilities of the quark Fermi liquid we employ the renormalization group methods described in 6 . The idea is quite simple. The low energy excitations of a degenerate Fermi systems are quasiparticles and holes in the vicinity of the Fermi surface, described by an effective action of the form

$$
S_{e f f}=\int d t d^{3} p \psi^{\dagger}\left(i \partial_{t}-\left(\epsilon(p)-\epsilon_{F}\right)\right) \psi+S_{i n t} .
$$

Quasiparticle interactions are represented by a tower of $2 n$ fermion operators. We can analyze the general structure of the interactions by studying the evolution of the corresponding operators as we successively integrate out modes closer and closer to the Fermi surface. The main result of this analysis is that, in general, four fermion, six fermion, and higher order interactions are suppressed as we approach the Fermi surface. This fixed point corresponds to Landau liquid theory. The only exceptions are the well known instabilities in the particle-particle and particle-hole channels. In the particle-particle channel the dominant operators correspond to two particles from opposite corners of the Fermi surface $\left(p_{F},-p_{F}\right)$ scattering into $\left(p_{F}^{\prime},-p_{F}^{\prime}\right)$. This kind of scattering leads to a logarithmic growth of the coupling constant as we approach the Fermi surface, and the BCS instability.

In QCD, a complete set of four fermion operators is given by

$$
\begin{array}{ll}
O_{L L}^{0}=\left(\bar{\psi}_{L} \gamma_{0} \psi_{L}\right)^{2}, & O_{L R}^{0}=\left(\bar{\psi}_{L} \gamma_{0} \psi_{L}\right)\left(\bar{\psi}_{R} \gamma_{0} \psi_{R}\right) \\
O_{L L}^{i}=\left(\bar{\psi}_{L} \gamma_{i} \psi_{L}\right)^{2}, & O_{L R}^{i}=\left(\bar{\psi}_{L} \vec{\gamma} \psi_{L}\right)\left(\bar{\psi}_{R} \vec{\gamma} \psi_{R}\right) .
\end{array}
$$

Each of these operators comes in two color structures, for example color symmetric and color anti-symmetric

$$
\left(\bar{\psi}^{a} \psi^{b}\right)\left(\bar{\psi}^{c} \psi^{d}\right)\left(\delta_{a b} \delta_{c d} \pm \delta_{a d} \delta_{b c}\right) .
$$

Nothing essentially new emerges from considering superficially different isospin structures, or different Dirac matrices. All such structures can be reduced to linear combinations of the basic ones (3), or their parity conjugates, by Fierz rearrangements. In total, we have to consider eight operators.

We should emphasize that we have restricted ourselves to massless QCD, and local operators invariant under the full $S U(3)_{L} \times S U(3)_{R} \times U(1)_{A}$ chiral symmetry. The extension to operators that break the anomalous $U(1)_{A}$ symmetry is discussed in 6 . Also, QCD at high density contains long range interactions due to unscreened magnetic gluon exchanges. The inclusion of long range forces is discussed in 20.11 and in section 6 below.

The operators (3) are renormalized by quark-quark scattering in the vicinity of the Fermi surface. This means that both incoming and outgoing quarks have

mbx: submitted to World Scientific on June 25, 2018 
momenta $\vec{p}_{1}, \vec{p}_{2} \simeq \pm \vec{p}$ and $\vec{p}_{3}, \vec{p}_{4} \simeq \pm \vec{q}$ with $|\vec{p}|,|\vec{q}| \simeq p_{F}$. We can take the external frequency to be zero. A graph with vertices $\Gamma_{1}$ and $\Gamma_{2}$ then gives

$$
G_{1} G_{2} I\left(\Gamma_{1}\right)_{i^{\prime} i}\left(\Gamma_{1}\right)_{k^{\prime} k}\left[-\left(\gamma_{0}\right)_{i j}\left(\gamma_{0}\right)_{k l}+\frac{1}{3}(\vec{\gamma})_{i j}(\vec{\gamma})_{k l}\right]\left(\Gamma_{2}\right)_{j j^{\prime}}\left(\Gamma_{2}\right)_{l l^{\prime}}
$$

with $I=\frac{i}{8 \pi^{2}} \mu^{2} \log \left(\Lambda_{I R} / \Lambda_{U V}\right)$. Here $\left[\Lambda_{I R}, \Lambda_{U V}\right]$ is the range of momenta that was integrated out. We will denote the density of states on the Fermi surface by $N=$ $\mu^{2} /\left(2 \pi^{2}\right)$ and the logarithm of the scale $t=\log \left(\Lambda_{I R} / \Lambda_{U V}\right)$. The renormalization group does not mix $L L$ and $L R$ operators, and it also does not mix different color structures. The evolution equations are

$$
\begin{aligned}
\frac{d\left(G_{0}^{L L}+G_{i}^{L L}\right)}{d t} & =-\frac{N}{3}\left(G_{0}^{L L}+G_{i}^{L L}\right)^{2}, \\
\frac{d\left(G_{0}^{L L}-3 G_{i}^{L L}\right)}{d t} & =-N\left(G_{0}^{L L}-3 G_{i}^{L L}\right)^{2}, \\
\frac{d\left(G_{0}^{L R}+3 G_{i}^{L R}\right)}{d t} & =0, \\
\frac{d\left(G_{0}^{L R}-G_{i}^{L R}\right)}{d t} & =-\frac{2 N}{3}\left(G_{0}^{L R}-G_{i}^{L R}\right)^{2} .
\end{aligned}
$$

In this basis the evolution equations are already diagonal. The coupling $G_{1}=$ $G_{0}^{L L}+G_{i}^{L L}$ evolves as

$$
G_{1}(t)=\frac{1}{1+(N / 3) G_{1}(0) t}
$$

with analogous results for the other operators. Note that the evolution starts at $t=0$ and moves towards the Fermi surface $t \rightarrow-\infty$. If the coupling is attractive at the matching scale, $G_{1}(0)>0$, it will grow during the evolution, and reach a Landau pole at $t_{c}=3 /\left(N G_{1}(0)\right)$. The corresponding energy scale is

$$
\Lambda_{I R}=\Lambda_{U V} \exp \left(-\frac{3}{N G_{1}(0)}\right) \text {. }
$$

This is the standard BCS result. The location of the pole is controlled by the initial value of the coupling and the coefficient in the evolution equation. If the initial coupling is negative, the coupling decreases during the evolution. The second operator in (6) has the largest coefficient and will reach the Landau pole first, unless the initial value is very small or negative. In that case, subdominant operators may determine the pairing.

The particular form of the operators that diagonalize the evolution equations can be made more transparent using a Fierz transformation. We find

$$
\begin{aligned}
O_{d o m} & =2\left(\psi_{L} C \psi_{L}\right)\left(\bar{\psi}_{L} C \bar{\psi}_{L}\right), \\
O_{\text {sub }, 1} & =\frac{1}{3}\left(\psi_{L} C \vec{\gamma} \psi_{R}\right)\left(\bar{\psi}_{R} C \vec{\gamma} \psi_{L}\right)+\ldots, \\
O_{\text {sub }, 2} & =\frac{4}{3}\left(\psi_{L} C \vec{\Sigma} \psi_{L}\right)\left(\bar{\psi}_{L} C \vec{\Sigma} \bar{\psi}_{L}\right), \\
O_{\text {mar }} & =\frac{1}{2}\left(\psi_{L} C \gamma_{0} \psi_{R}\right)\left(\bar{\psi}_{R} C \gamma_{0} \psi_{L}\right)+\ldots,
\end{aligned}
$$

mbx: submitted to World Scientific on June 25, 2018 
where we have ordered the operators according to the size of the coefficient in the evolution equations. We find that the dominant operator corresponds to pairing in the scalar diquark channel, while the subdominant operators contain vector diquarks. Note that from the evolution equation alone we cannot decide what the preferred color channel is. To decide this question, we must invoke the fact that "reasonable" interactions, like one gluon exchange, are attractive in the color antisymmetric repulsive in the color symmetric channel. This can be seen from the identity

$$
(\vec{\lambda})^{a b}(\vec{\lambda})^{c d}=\frac{2}{3}\left(\delta^{a b} \delta^{c d}+\delta^{a d} \delta^{b c}\right)-\frac{4}{3}\left(\delta^{a b} \delta^{c d}-\delta^{a d} \delta^{b c}\right) .
$$

If the color wave function is anti-symmetric, the Pauli exclusion principle fixes the isospin wave function to be anti-symmetric as well. The dominant operator does not distinguish between scalar and pseudoscalar diquarks. This degeneracy is lifted by operators that break the axial baryon number symmetry. These operators are associated with instantons. As we will discuss in more detail in section f, instantons favor scalar diquarks over pseudoscalar diquarks.

\section{The effective potential}

The form of the dominant operator indicates the existence of potential instabilities, but does not itself indicate how they are resolved. In this section we wish to introduce a simple energy functional that captures the essential dynamics of QCD at high baryon density. We shall use this functional in order to analyze the ground state of QCD with different numbers of colors and flavors.

The dominant coupling is a color and flavor ant-symmetric interaction of the form

$$
\mathcal{L}=G\left(\delta^{a c} \delta^{b d}-\delta^{a d} \delta^{b c}\right)\left(\delta_{i k} \delta_{j l}-\delta_{i l} \delta_{j k}\right)\left(\psi_{i}^{a} C \gamma_{5} \psi_{j}^{b}\right)\left(\bar{\psi}_{k}^{c} C \gamma_{5} \bar{\psi}_{l}^{d}\right)
$$

where $a, b, \ldots$ are color indices and $i, j \ldots$ are flavor indices. In the following, we shall use the notation $\mathcal{K}_{i j k l}^{a b c d}$ for the color-flavor structure of the interaction.

In order to determine the structure of the ground state we have to calculate the grand canonical potential of the system for different trial states. Since the interaction is attractive in s-wave states, it seems clear that the dominant order parameter is an s-wave, too. We then only have to study the color-flavor structure of the primary condensate. We assume that the condensate takes the form

$$
\left\langle\psi_{i}^{a} C \gamma_{5} \psi_{j}^{b}\right\rangle=\phi_{i j}^{a b} .
$$

$\phi_{i j}^{a b}$ is a $N_{f} \times N_{f}$ matrix in flavor space and a $N_{c} \times N_{c}$ matrix in color space.

We calculate the effective potential using the bosonization method. For this purpose, we introduce collective fields $\Delta_{i j}^{a b}$ and $\bar{\Delta}_{i j}^{a b}$ with the symmetries of the order parameter (18). We add to the fermionic action a term $G^{-1} \mathcal{K}_{i j k l}^{a b c d} \Delta_{i j}^{a b} \bar{\Delta}_{k l}^{c d}$ and integrate over the dummy variables $\Delta_{i j}^{a b}$ and $\bar{\Delta}_{i j}^{a b}$. We then shift the integration variables to eliminate the interaction term (17). So far, no approximations have been made. We now assume that the collective fields are slowly varying, and that $\Delta_{i j}^{a b}$ can be replaced by a constant. In this case, we can perform the integration

mbx: submitted to World Scientific on June 25, 2018 
over the fermionic fields and determine the grand canonical potential as a function of the gap matrix $\Delta_{i j}^{a b}$.

The integration over the fermions is performed using the Nambu-Gorkov formalism. We introduce a two component field $\Psi=\left(\psi, \bar{\psi}^{T}\right)$. The inverse quark propagator takes the form

$$
S^{-1}(q)=\left(\begin{array}{cc}
\not q+\not h-m & \mathcal{K} \cdot \bar{\Delta} \\
\mathcal{K} \cdot \Delta & (\not q-\not L+m)^{T}
\end{array}\right) .
$$

The grand canonical potential is now given by

$$
\Omega(\Delta)=\frac{1}{2} \operatorname{Tr}[\log (S)]+\frac{1}{G} \Delta \cdot \mathcal{K} \cdot \bar{\Delta} .
$$

In order to evaluate the logarithm, we have to diagonalize the mass matrix $\mathcal{M}=$ $\mathcal{K} \cdot \Delta$. Let us denote the corresponding eigenvalues by $\delta_{\rho}\left(\rho=1, \ldots, N_{c} N_{f}\right)$. These are the physical gaps of the $N_{f} N_{c}$ fermion species. The $\operatorname{tr} \log (S)$ term has an ultraviolet divergence. This divergence can be regularized in a way that is consistent with the renormalization group approach 12 . We find

$$
\Omega_{r e n}(\Delta)=\sum_{\rho}\left\{-\frac{\mu^{2}}{4 \pi^{2}} \delta_{\rho}^{2}\left(\log \left(\frac{\delta_{\rho}}{\xi}\right)-1\right)+\frac{4}{G(\xi)} \delta_{\rho}^{2}\right\} .
$$

Here, $\xi$ is a renormalization scale. The grand potential is independent of $\xi$, since the scale dependence of the first term is canceled by the scale dependence of the coupling constant $G$. The coupling constant satisfies the renormalization group equation discussed above.

\section{Superconductivity in QCD with $N_{c}$ colors and $N_{f}$ flavors}

From the minima of the effective potential we can now determine the groundstate properties in a theory with $N_{c}$ colors and $N_{f}$ flavors. The simplest case is QCD with two colors and two flavors. In this case, we have

$$
\Delta_{i j}^{a b}=\Delta \epsilon^{a b} \epsilon_{i j}, \quad\left(N_{c}=N_{f}=2\right) .
$$

This order parameter is a color and flavor singlet. This would seem to indicate that no symmetries are broken, but this conclusion is not correct. QCD with two colors has a particle-anti-particle (Pauli-Gürsey) symmetry which enlarges the flavor symmetry of the massless theory from $S U(2) \times S U(2)$ to $S U(4)$. Spontaneous chiral symmetry breaking by a non-zero quark condensate $\langle\bar{\psi} \psi\rangle$ corresponds to the symmetry breaking pattern $S U(4) \rightarrow S p(4)$. The coset $S U(4) / S p(4)=S^{5}$, so there are 5 Goldstone bosons. These Goldstone bosons are the three pions, the scalar diquark, and its anti-particle. Under the Pauli-Gürsey symmetry the chiral condensate $\langle\bar{\psi} \psi\rangle$ is equivalent to the diquark condensate $\left\langle\epsilon^{a b} \epsilon_{i j} \psi_{i}^{a} C \gamma_{5} \psi_{j}^{b}\right\rangle$. In the diquark condensed phase, the five Goldstone bosons are the three pions, the sigma, and the anti-scalar diquark.

Both a quark mass term and a chemical potential break the $S U(4)$ symmetry. If the chemical potential is zero, but the quark mass is not, then the theory has a non-zero chiral condensate. If the chemical potential is increased there is a phase

mbx: submitted to World Scientific on June 25, 2018 
transition at a critical chemical potential $\mu_{c} \simeq m_{\pi} / 2$. For $\mu>\mu_{c}$, the order parameter rotates from the chiral condensate to the diquark condensate. QCD with two colors is discussed in more detail in 23.14.

We would now like to discuss real QCD with three colors. The case of only one flavor is special, since in this case a color anti-symmetric condensate cannot be a spin singlet. We therefore expect condensation to take place in a spin- 1 channel. This problem is of some relevance for the behavior of real QCD with two light and one intermediate mass flavor. For $m_{s}$ larger than some critical value, QCD has a phase with separate pairing in the $u d$ and $s$ sectors 15,16 . Here, we will not discuss $N_{f}=1 \mathrm{QCD}$ any further.

In the case of two flavors, the order parameter

$$
\Delta_{i j}^{a b}=\Delta \epsilon^{3 a b} \epsilon_{i j}, \quad\left(N_{c}=3, N_{f}=2\right),
$$

breaks color $S U(3) \rightarrow S U(2)$. The chiral $S U(2)_{L} \times S U(2)_{R}$ symmetry remains unbroken. At this level, the Fermi surfaces of the up and down quarks of the third color remain intact. Subleading interactions can generate a gap for these states. The exact nature of this gap is hard to determine, even in the limit of very large chemical potential.

The mostinteresting case is the situation for three colors and three flavors. As shown in 15.16, these results are relevant to real QCD as long as $m_{s}^{2}<4 \mu \Delta$. In this case the order parameter takes the form

$$
\Delta_{i j}^{a b}=\Delta\left(\delta_{i}^{a} \delta_{j}^{b}-\delta_{i}^{b} \delta_{j}^{a}\right), \quad\left(N_{c}=N_{f}=3\right) .
$$

This is the color-flavor locked phase suggested in 7 . Both color and flavor symmetry are completely broken, but there are eight combinations of color and flavor symmetries that generate unbroken global symmetries. This is obvious from the result (24). We can always undo a flavor rotation by an $S U(3)$ matrix $U$ by performing a subsequent color rotation with the matrix $U^{*}$. Note that since color is a vector-like symmetry, the remaining flavor symmetry is vector-like also. The symmetry breaking pattern is

$$
S U(3)_{L} \times S U(3)_{R} \times U(1)_{V} \rightarrow S U(3)_{V} .
$$

This is exactly the same symmetry breaking that QCD exhibits at low density. This opens the interesting possibility that in QCD with three flavors, the low and high density phases might be continuously connected 18 . We will discuss this phase in more detail in section 7 .

The phase structure of QCD-like theories with more than three flavors was studied in 1 . The main result is that the general structure found in the case of three flavors extends to more than three flavors. The order parameter involves a coupling between color and flavor degrees of freedom and the chiral $S U\left(N_{f}\right)_{L} \times S U\left(N_{f}\right)_{R}$ symmetry is broken down to a vector-like subgroup. In general, however, this subgroup is not the full flavor $S U\left(N_{f}\right)_{V}$ flavor symmetry that is present at zero density. In the case of four flavors, for example, one finds $S U(4)_{L} \times S U(4)_{R} \rightarrow$ $O(4)_{V}$.

mbx: submitted to World Scientific on June 25, 2018 


\section{Instantons and Color Superconductivity}

The first quantitative estimate of the superconducting gap was given by Bailin and Love 1 . They assumed that the gap is generated by perturbative one-gluon exchange. In this case one has to deal with the problem that the quark-quark scattering amplitude is singular at small angles. Bailin and Love assumed that in a cold quark liquid both the electric and magnetic interactions are screened at some scale $\Lambda$. In this case, the gap is on the order of $\Delta \sim \mu \exp \left(-6 \pi^{2} /\left(g^{2} L\right)\right)$, where $L=\log \left(\mu^{2} / \Lambda^{2}\right)$ depends logarithmicly on the screening scale. This result implies that $\Delta$ is quite small, unless we are willing to consider very large coupling constants, $\alpha_{s}=g^{2} /(4 \pi)>1$. Bailin and Love concluded that, most likely, $\Delta / \mu \sim$ $O\left(10^{-3}\right)$ for chemical potentials $\mu<1 \mathrm{GeV}$. We will see in the next section that the correct result for the perturbative gap is $\Delta \sim \mu g^{-5} \exp \left(-3 \pi^{2} /(\sqrt{2} g)\right)$, which is parametrically bigger than the result of Bailin and Love. Nevertheless, it remains unclear whether perturbation theory is applicable for chemical potentials that are of practical interest.

After the work of Bailin and Love, there was practically no interest in quark superconductivity until two groups independently pointed out that non-perturbative effects, instantons, can generate gaps that are much larger, on the order of $100 \mathrm{MeV}$ at densities a few times nuclear matter density 13.20 . In general it was realized that any kind of effective quark interaction which is consistent with the magnitude of the chiral condensate and general features of the hadron spectrum at zero density will lead to gaps in that range. As we saw above, this is most obvious in the case of two colors, where the diquark condensate is related to the chiral condensate by a symmetry.

Instantons are classical solutions of the euclidean Yang-Mills field equations, describing tunneling events between topologically different vacuum configurations. Instantons are the strongest non-perturbative fluctuations in the QCD vacuum; when lattice gauge configurations are made somewhat more smooth, it is instantons one mainly finds, see 21 for a recent review. Instantons have a dramatic effect on quarks. In the background field of an instanton, the Dirac operator has an exact, chiral, zero mode. This zero mode is connected with the chiral anomaly, but it also plays an important role in connection with spontaneous chiral symmetry breaking. There is evidence that the quark condensate can be understood as a collective state built from instanton zero modes. These zero modes, just like the chiral anomaly, continue to exist at non-zero chemical potential. In this case, the zero modes lead to a pairing interaction, and superconductivity.

This is most easily discussed in terms of effective interactions. In the case of two flavors, the effect of zero modes on the propagation of quarks can be encoded in a four fermion interaction

$$
\begin{aligned}
\mathcal{L}=g \frac{1}{4\left(N_{c}^{2}-1\right)}\{ & \frac{2 N_{c}-1}{2 N_{c}}\left[\left(\bar{\psi} \mathcal{F}^{\dagger} \tau_{\alpha}^{-} \mathcal{F} \psi\right)^{2}+\left(\bar{\psi} \mathcal{F}^{\dagger} \gamma_{5} \tau_{\alpha}^{-} \mathcal{F} \psi\right)^{2}\right] \\
& \left.+\frac{1}{4 N_{c}}\left(\bar{\psi} \mathcal{F}^{\dagger} \sigma_{\mu \nu} \tau_{\alpha}^{-} \mathcal{F} \psi\right)^{2}\right\}
\end{aligned}
$$

where $N_{c}$ is the number of colors, $\tau^{-}=(\vec{\tau}, i)$ is an isospin matrix, and $\mathcal{F}$ is a form factor that reflects the zero mode wave function 2324 . From this interaction we can

mbx: submitted to World Scientific on June 25, 2018 


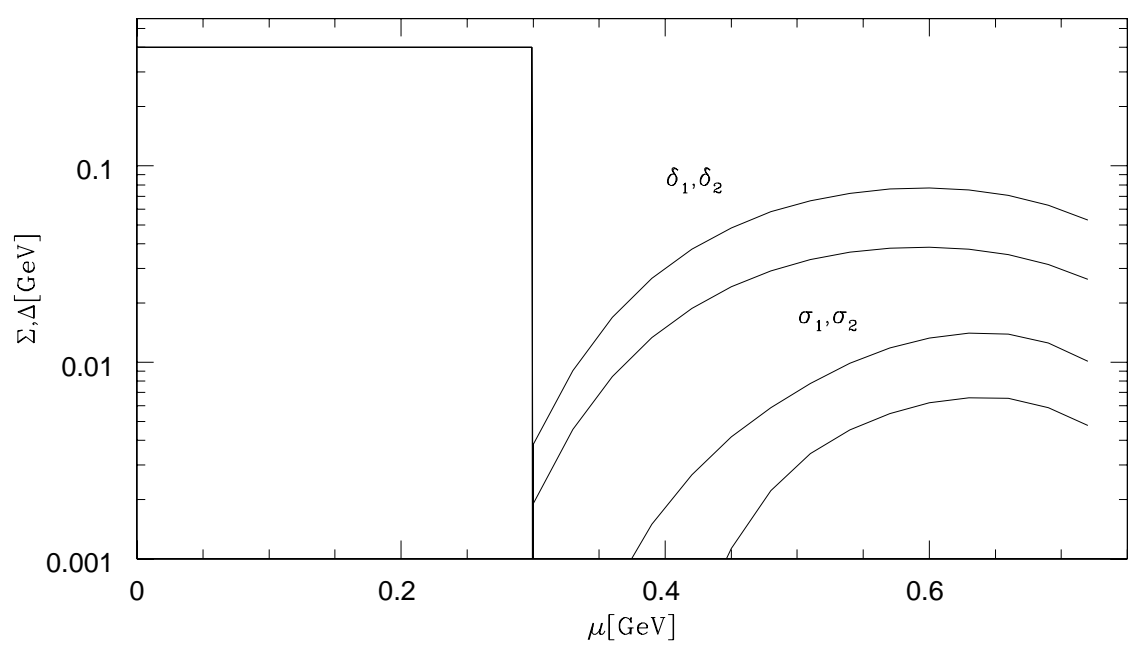

Figure 1. Quark constituent mass and superconducting gap from a mean field calculation in $N_{f}=3$ QCD.

directly read off the effects of instantons in different quark-anti-quark channels. In the pseudoscalar channel the interaction combines attraction for isospin 1 (pion) channel with repulsion for isospin $0\left(\eta^{\prime}\right)$. Similarly, one finds attraction in the scalar isospin-0 $(\sigma)$ channel (responsible for spontaneous chiral symmetry breaking) together with repulsion in the scalar isospin-1 channel $\left(a_{0}\right)$.

Using simple Fierz identities, we can construct the effective interaction for color antisymmetric $[\overline{3}]$ and symmetric $[6]$ diquarks. The result is

$$
\begin{aligned}
& \mathcal{L}_{d i q}=\frac{g}{8 N_{c}^{2}}\left\{-\frac{1}{N_{c}-1}\right. {\left[( \psi ^ { T } \mathcal { F } ^ { T } C \tau _ { 2 } \lambda _ { A } ^ { a } \mathcal { F } \psi ) \left(\bar{\psi} \mathcal{F}^{\dagger} \tau_{2} \lambda_{A}^{a} C \mathcal{F}^{*} \bar{\psi}^{T}\right.\right.} \\
&+\left.\left(\psi^{T} \mathcal{F}^{T} C \tau_{2} \lambda_{A}^{a} \gamma_{5} \mathcal{F} \psi\right)\left(\bar{\psi} \mathcal{F}^{\dagger} \tau_{2} \lambda_{A}^{a} \gamma_{5} C \mathcal{F}^{*} \bar{\psi}^{T}\right)\right] \\
&\left.+\frac{1}{2\left(N_{c}+1\right)}\left(\psi^{T} \mathcal{F}^{T} C \tau_{2} \lambda_{S}^{a} \sigma_{\mu \nu} \mathcal{F} \psi\right)\left(\bar{\psi} \mathcal{F}^{\dagger} \tau_{2} \lambda_{S}^{a} \sigma_{\mu \nu} C \mathcal{F}^{*} \bar{\psi}^{T}\right)\right\},
\end{aligned}
$$

where $\tau_{2}$ is the anti-symmetric Pauli matrix, and $\lambda_{A, S}$ are the anti-symmetric and symmetric color generators. In the color $[\overline{3}]$ channel, the interaction is attractive for scalar $\left(\psi^{T} C \gamma_{5} \psi\right)$ diquarks, and repulsive for pseudoscalar $\left(\psi^{T} C \psi\right)$ diquarks.

We can now use this interaction in order to study the phase structure of $N_{f}=2$ matter 22.23.24. This is most easily done in the mean field approximation. At zero chemical potential chiral condensation is favored over diquark condensation, simply because the corresponding coupling constant is larger. At finite chemical potential chiral condensation is suppressed because quark loops are Pauli blocked. Quarkquark pairing, on the other hand, is enhanced because the density of states on the Fermi surface grows. As a result we find a first order transition from a phase with chiral symmetry breaking to a superconducting phase. As an example, we show the

mbx: submitted to World Scientific on June 25, 2018 
results of a somewhat more sophisticated calculation 24 in $N_{f}=3 \mathrm{QCD}$ in figure 1. The instanton density was fixed in order to reproduce a constituent quark mass $M=400 \mathrm{MeV}$ at zero chemical potential. In this case, we find the transition to the superconducting phase at a quark chemical potential $\mu_{c} \simeq 300 \mathrm{MeV}$. In QCD with three flavors there is a flavor singlet and a flavor octet gap in the high density phase. The singlet gap peaks at around $80 \mathrm{MeV}$. As we will discuss in more detail below, chiral symmetry remains broken in the high density phase. There are two types of constituent quark masses in the high density phase, but both of them are small, $M<10 \mathrm{MeV}$. We should stress that the mean field description provides a very simple minded picture of the phase transition. The critical chemical potential is very small, and there is no nuclear matter phase in which quarks are clustered into nucleons.

\section{Superconductivity from perturbative one-gluon exchange}

If the chemical potential is very large, asymptotic freedom implies that the coupling is weak. In this case, the gap can be calculated in weak coupling perturbation theory. The result $\Delta \sim \mu g^{-5} \exp \left(-3 \pi^{2} /(\sqrt{2} g)\right)$ mentioned above was first derived by Son using renormalization group arguments. It has since then been rederived in a number of works 25,26 , 28 2 . Here we will follow 25 and concentrate on the $N_{f}=2$ order parameter (23). The gap matrix has the form

$$
\Delta_{i j}^{a b}(q)=\left(\lambda_{2}\right)^{a b}\left(\tau_{2}\right)_{i j} C \gamma_{5}\left(\Delta_{1}\left(q_{0}\right) \frac{1}{2}(1+\vec{\alpha} \cdot \hat{q})+\Delta_{2}\left(q_{0}\right) \frac{1}{2}(1-\vec{\alpha} \cdot \hat{q})\right) .
$$

This decomposition is useful in order to discuss the weak coupling limit and make the gauge invariance of the result manifest. In weak coupling, we can replace $\vec{\alpha} \cdot \hat{q} \rightarrow 1$ and only $\Delta_{1}$ produces a gap on the Fermi surface. We have kept the dependence of the gap on the frequency. We will justify the necessity for this below. In condensed matter physics, keeping retardation effects in the gap equation is referred to as Eliashberg theory.

We calculate the gap by solving the Dyspn-Schwinger equation for the quark self-energy in the Nambu-Gorkov formalism

$$
\Sigma(k)=-i g^{2} \int \frac{d^{4} q}{(2 \pi)^{4}} \Gamma_{\mu}^{a} S(q) \Gamma_{\nu}^{b} D_{\mu \nu}^{a b}(q-k) .
$$

Here, $\Sigma(k)=-\left(S^{-1}(k)-S_{0}^{-1}(k)\right)$ is the proper self energy, $\Gamma_{\mu}^{a}$ is the quark-gluon vertex and $D_{\mu \nu}^{a b}(q-k)$ is the gluon propagator. The off-diagonal components of (29) determine the gap, while the diagonal components fix the wave function renormalization. Since the fermion momenta are large we will ignore wave function renormalization. For the same reason, we can also ignore vertex corrections.

The gluon momentum, however, can become soft and corrections to the gluon propagator have to be taken into account. The gluon propagator in a general covariant gauge is

$$
D_{\mu \nu}(q)=\frac{P_{\mu \nu}^{T}}{q^{2}-G}+\frac{P_{\mu \nu}^{L}}{q^{2}-F}-\xi \frac{q_{\mu} q_{\nu}}{q^{4}}
$$

mbx: submitted to World Scientific on June 25, 2018 
where $P_{\mu \nu}^{T, L}$ are transverse and longitudinal projectors and $G, F$ are functions of energy and momentum. $\xi$ is a gauge parameter that should not appear in any physical result.

We can derive two coupled equations for the gap parameters $\Delta_{1,2}\left(p_{0}\right)$. In the weak coupling limit, we find that only terms involving $\Delta_{1}$ have a singularity on the Fermi surface. We can therefore drop $\Delta_{2}$. The resulting equation for $\Delta_{1}$ is independent of the gauge parameter. We find

$$
\begin{aligned}
\Delta\left(p_{0}\right)=\frac{g^{2}}{12 \pi^{2}} \int d q_{0} \int d \cos \theta & \left(\frac{\frac{3}{2}-\frac{1}{2} \cos \theta}{1-\cos \theta+\left(G+\left(p_{0}-q_{0}\right)^{2}\right) /\left(2 \mu^{2}\right)}\right. \\
+ & \left.\frac{\frac{1}{2}+\frac{1}{2} \cos \theta}{1-\cos \theta+\left(F+\left(p_{0}-q_{0}\right)^{2}\right) /\left(2 \mu^{2}\right)}\right) \frac{\Delta\left(q_{0}\right)}{\sqrt{q_{0}^{2}+\Delta\left(q_{0}\right)^{2}}} .
\end{aligned}
$$

The integral over $\cos \theta$ is dominated by small $\theta$, corresponding to almost collinear scattering. It is therefore important to take medium modifications of the gluon propagator at small momenta into account. For $\vec{q} \rightarrow 0$ and to leading order in perturbation theory we have

$$
F=2 m^{2}, \quad G=\frac{\pi}{2} m^{2} \frac{q_{0}}{|\vec{q}|},
$$

with $m^{2}=N_{f} g^{2} \mu^{2} /\left(4 \pi^{2}\right)$. In the longitudinal part, $m_{D}^{2}=2 m^{2}$ is the familiar Debye screening mass. In the transverse part, there is no screening of static modes, but non-static modes are modes are dynamically screened due to Landau damping. In our case, typical frequencies are on the order of the gap, $q_{0} \simeq \Delta$. This means that the electric part of the interaction is screened at $q_{E} \simeq m_{D}^{1 / 2}$ whereas the magnetic interaction is screened at $q_{M} \simeq\left(\pi / 4 \cdot m_{D}^{2} \Delta\right)^{1 / 3}$. Asymptotically, $q_{M} \ll q_{E}$, and magnetic gluon exchange dominates over electric gluon exchange.

We can now perform the angular integral and find

$$
\Delta\left(p_{0}\right)=\frac{g^{2}}{18 \pi^{2}} \int d q_{0} \log \left(\frac{b \mu}{\left|p_{0}-q_{0}\right|}\right) \frac{\Delta\left(q_{0}\right)}{\sqrt{q_{0}^{2}+\Delta\left(q_{0}\right)^{2}}},
$$

with $b=256 \pi^{4}\left(2 / N_{f}\right)^{5 / 2} g^{-5}$. We can now see why it was important to keep the frequency dependence of the gap. Because the collinear divergence is regulated by dynamic screening, the gap equation depends on $p_{0}$ even if the frequency is small. We can also understand why the gap scales as $\exp (-c / g)$. The collinear divergence leads to a gap equation with a double-log behavior. Qualitatively

$$
1 \sim \frac{g^{2}}{18 \pi^{2}}\left[\log \left(\frac{\mu}{\Delta}\right)\right]^{2}
$$

from which we conclude that $\Delta \sim \exp (-c / g)$. The approximation (34) is not sufficiently accurate to determine the correct value of the constant $c$. For this purpose, we have to solve the integral equation (33) This can be done by converting the integral equation into a differential equation 10 . In the weak coupling limit, an approximate solution is given by

$$
\Delta\left(p_{0}\right) \simeq \Delta_{0} \sin \left(\frac{g}{3 \sqrt{2} \pi} \log \left(\frac{b \mu}{p_{0}}\right)\right), \quad p_{0}>\Delta_{0},
$$

mbx: submitted to World Scientific on June 25, 2018 
with $\Delta_{0}=b \mu \exp \left(-3 \pi^{2} /(\sqrt{2} g)\right)$. The final result for the magnitude of the gap on the Fermi surface is

$$
\Delta_{0} \simeq 256 \pi^{4}\left(2 / N_{f}\right)^{5 / 2} \mu g^{-5} \exp \left(-\frac{3 \pi^{2}}{\sqrt{2} g}\right) .
$$

We should emphasize that, strictly speaking, this result contains only an estimate of the pre-exponent. This estimate is obtained by collecting the leading logarithms from electric and magnetic gluon exchanges. We also note that the result shows that the perturbative calculation is self-consistent. Asymptotically, the factor $\mu$ in the pre-exponent overwhelms the exponential suppression factor $\exp (-c / g)$ and $\Delta \gg \Lambda_{Q C D}$. This implies that the magnetic screening scale $q_{M} \sim\left(g^{2} \mu^{2} \Delta\right)^{1 / 3} \gg$ $\Lambda_{Q C D}$ and the perturbative result for the gluon polarization function is reliable.

For chemical potentials $\mu<1 \mathrm{GeV}$, the coupling constant is not small and the applicability of perturbation theory is in doubt. It is known that at finite temperature the convergence properties of the perturbative expansion are extremely poor. It has been argued that the situation at non-zero chemical potential might be better 26. The superconducting gap, however, is dominated by collinear exchanges. For $\mu<1 \mathrm{GeV}$ we have $q_{M} \simeq \Lambda_{Q C D}$ and the gap is mainly determined by very small momenta. If we ignore this problem and calculate the gap at chemical potentials on the order of $500 \mathrm{MeV}$, we find $\Delta \simeq 100 \mathrm{MeV}$. It is gratifying to see that this result is quite close to the low-density estimate based on instantons or other effective interactions.

\section{Color-Flavor Locking and Quark-Hadron duality in QCD with three flavors}

In this section we wish to study the color-flavor locked phase in QCD with $N_{f}=$ 3 flavors in somewhat more detail. In the limit where all quarks are massless the underlying theory has the continuous symmetry group $S U(3)_{C} \times S U(3)_{L} \times$ $S U(3)_{R} \times U(1)_{B}$. In the color-flavor locked phase the symmetry is broken following the pattern

$$
S U(3)_{C} \times S U(3)_{L} \times S U(3)_{R} \times U(1)_{B} \rightarrow S U(3)_{C+L+R} \times Z_{2} .
$$

The breaking of local color symmetry implies that all the gluons acquire mass, according to the Higgs effect. There are no long-range interactions. At low energy, there is no direct signature for the color degree of freedom. Color is, in this sense, confined.

As explained in section 1 , chiral symmetry is broken because color, being a vector-like interaction, locks left handed flavor rotation to right handed flavor rotations. This is an unusual mechanism for chiral symmetry breaking. The condensates $\left\langle\psi_{L} \psi_{L}\right\rangle$ and $\left\langle\psi_{R} \psi_{R}\right\rangle$ of left and right handed quarks are quite separate. Indeed, unless we include instantons, they are not even phase coherent. Nevertheless, because both are locked to color they are thereby locked to one another.

Superconductivity breaks the $U(1)$ of baryon number. This might seem disconcerting at first, but the total baryon number of a finite sample is still conserved. The meaning of baryon number violation is that there is a Goldstone mode, the

mbx: submitted to World Scientific on June 25, 2018 
phonon, that corresponds to small fluctuations in the local baryon density. The macroscopic consequence of this fact is easy transport of baryon number, or superfluidity. We can construct a gauge invariant order parameter for superfluidity by convoluting several of the primary, diquark, condensates:

$$
\chi=\left\langle\epsilon^{a b c} \epsilon_{i j k} \phi_{i}^{a} \phi_{j}^{b} \phi_{k}^{c}\right\rangle, \quad \phi_{i}^{a}=\epsilon^{a b c} \epsilon_{i j k} \psi_{j}^{b} C \gamma_{5} \psi_{k}^{c} .
$$

We note that this order parameter carries the quantum numbers of a di-lambda, or the hypothetical H-dibaryon.

A diquark condensate breaks baryon number but there is a residual $Z_{2}$ symmetry that corresponds to flipping the sign of the quark fields. The primary condensate also leaves an axial $Z_{2}$ symmetry, but in QCD this symmetry is broken by instantons. At large chemical potential instantons and other semi-classical fluctuations are suppressed, and the axial $Z_{2}$ symmetry is almost exact. One consequence of this result is that the ordinary chiral condensate $\langle\bar{\psi} \psi\rangle$ is strongly suppressed, whereas its square $\left\langle(\bar{\psi} \psi)^{2}\right\rangle$ is not small, proportional to the primary diquark condensate squared 19 .

It is interesting to consider how the photon couples to color-flavor locked matter. The original electromagnetic gauge invariance is broken, but there is a combination of the original electromagnetic gauge symmetry and a color transformation which leaves the condensate invariant. The original photon $\gamma$ couples with strength $e$ to the flavor matrix $\operatorname{diag}(2 / 3,-1 / 3,-1 / 3)$. There is a diagonal gluon $G$ which couples with strength $g$ to the color matrix $\operatorname{diag}(-2 / 3,1 / 3,1 / 3)$. Then the combination

$$
\tilde{\gamma}=\frac{g \gamma+e G}{\sqrt{e^{2}+g^{2}}}
$$

leaves the color-flavor locked condensate invariant. In the color superconducting medium it represents the physical photon. This photon couples to electrons with coupling $e g / \sqrt{e^{2}+g^{2}}$, which is the unit of charge in the color-flavor locked phase. The coupling to quarks receives contributions from both the color and the flavor charges. It is easy to see that, in units of the electron charge, these contributions always add up to \pm 1 or 0 . The same is true for gluons, too.

We are now in a position to examine the low energy excitations in the color-flavor locked phase. The spontaneous breaking of global chiral $S U(3)_{L} \times S U(3)_{R}$ brings with it an octet of pseudoscalar Nambu-Goldstone bosons. In addition to that there is a massless phonon, corresponding to the spontaneous breaking of $U(1)_{B}$. If the density is very high, there is also an anomalously light pseudo-Goldstone boson associated with the $U(1)_{A}$ symmetry.

In addition to the Goldstone modes, there are also excitations directly derived from the original quark and gluon modes. The quarks form an octet and a singlet of spin $1 / 2$ particles under the residual $S U(3)_{V}$. There is an energy gap for the production of quark pairs. Dynamical calculations show that this gap is larger for the singlet than for the octet. This means that the states in the octet are true long-lived quasiparticles, since there is nothing for them to decay into. We can think of these states as baryons, since baryon number is only conserved modulo $2 / 3$. The gluon fields form an octet of spin 1 bosons under the $S U(3)_{V}$ symmetry. They acquire a mass by the Meissner-Higgs phenomenon.

mbx: submitted to World Scientific on June 25, 2018 
The universal features of the color-flavor locked state: confinement, chiral symmetry breaking down to vector $S U(3)$, and superfluidity, are just what one would expect for $N_{f}=3 \mathrm{QCD}$ at low density. We have now seen that the same is true for the spectrum of low lying non-Goldstone excitations: There is an octet and singlet of baryons, and an octet of vector mesons. This means that there is no sharp distinction between the low-density, hadronic, phase and the high-density, quark, phase. In particular, there need not be a phase transition separating the high and low density phases of $N_{f}=3$ QCD. Furthermore, we have realized and old goal of QCD: we have obtained a weak coupling, but non-perturbative, description of hadronic degrees of freedom in terms of quarks and gluons.

\section{Chiral density waves}

Throughout this contribution we have assumed that the dominant instability at large density corresponds to the formation of particle-particle (or hole-hole) pairs. This is a very reasonable assumption, because only this kind of pairing occurs for arbitrarily weak coupling and uses the whole Fermi surface coherently. Nevertheless, in strong coupling, or if superconductivity is suppressed, other forms of pairing may take place. Obvious candidates are the formation of larger clusters (nucleons, fourquark states, dibaryons) or particle-hole pairing 29,30.31.

Particle-hole pairing is characterized by an order parameter of the form

$$
\langle\bar{\psi}(x) \psi(y)\rangle=\exp (i \vec{p} \cdot(\vec{x}+\vec{y})) \Sigma(x-y),
$$

where $|\vec{p}|=p_{F}$ is a vector on the Fermi surface. This state describes a chiral density wave. It was first suggested in 29 as the ground state of QCD at large chemical potential and large $N_{c}$. The color factors for particle-particle and particle-hole scattering are

$$
c=\frac{N_{c}+1}{2 N_{c}} \quad(p p), \quad c=\frac{N_{c}^{2}-1}{2 N_{c}} \quad(p h),
$$

suggesting that particle-particle pairing, and superconductivity, is suppressed at large $N_{c}$. Particle-hole pairing is not suppressed, but suffers from the fact that a direction is singled out and only a small part of the Fermi surface is used. At large $N_{c}$, however, screening due to fermions is weak and the perturbative one-gluon exchange interaction is even more strongly dominated by collinear exchanges. This favors the particle-hole instability.

Screening was ignored in the original work of 29 , but is taken into account in the more recent investigations 30,31. The main conclusion is that, in weak coupling, the chiral density wave instability requires very large $N_{c} \gg 3$. We have verified this fact from numerical solutions of the chiral density wave gap equation. In perturbative QCD, the gap equation reads 31

$$
\begin{aligned}
\Sigma\left(p_{\|}\right)=c \frac{g^{2}}{12 \pi^{2}} \int d q_{\|} & \left\{\log \left(1+\frac{\Lambda_{\perp}^{3}}{\left(p_{\|}-q_{||}\right)^{3}+\frac{\pi}{4} m_{D}^{2}\left|p_{\|}-q_{\| \mid}\right|}\right)\right. \\
+ & \left.\frac{3}{2} \log \left(1+\frac{\Lambda_{\perp}^{2}}{\left(p_{\|}-q_{||}\right)^{2}+m_{D}^{2}}\right)\right\} \frac{\Sigma\left(q_{\|}\right.}{\sqrt{q_{\|}^{2}+\Sigma\left(q_{||}\right)^{2}}} .
\end{aligned}
$$

mbx: submitted to World Scientific on June 25, 2018 


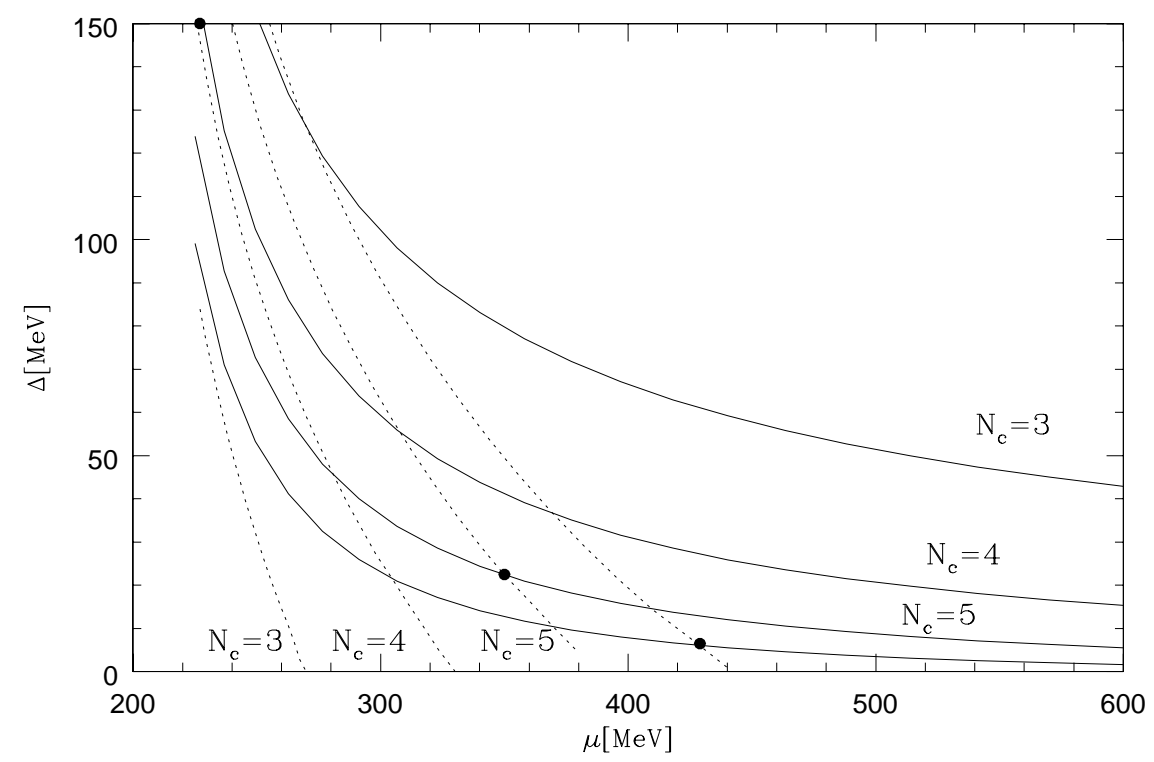

Figure 2. BCS (solid line) and chiral density wave (dashed line) gaps from a perturbative calculation in QCD with $N_{c}$ colors and $N_{f}=2$ flavors.

This gap equation is very similar to the BCS gap equation (33), except that the color factor is different, and that the angular integration is cut off at a scale $\Lambda_{\perp}=\sqrt{2 \mu q_{\|}}$. This fact reflects the one-dimensional nature of the chiral density wave.

Numerical results for both the BCS and chiral density wave (CDW) gap are shown in figure 2. We find that for moderate $N_{c}$, in particular in the case $N_{c}=$ 3 , the chiral density wave instability only exists for very small $\mu$ and very large coupling. In the case of $N_{c}=3$, the CDW gap is never bigger than the BCS gap, even if the coupling is big.

These results are not really quantitative. Only for very large $N_{c}$ does the chiral density wave instability occur in weak coupling where the perturbative analysis is appropriate. Nevertheless, our results illustrate the point that in the real world, with $N_{c}=3$, superconductivity is the dominant instability even at moderate chemical potential. Using more realistic interactions in the strong coupling regime is not likely to change this situation, since other interactions, like instantons, will typically lack the strong collinear enhancement of the one gluon exchange interaction. In addition to that, the true ground state is not determined by the size of the gap, but by the magnitude of the condensation energy. Since the chiral density wave state does not use the whole Fermi surface, the condensation energy is only of the order $\mu \Sigma^{3}$, as compared to $\mu^{2} \Delta^{2}$ in the case of superconductivity. 


\section{Conclusion: The many phases of QCD}

We would like to conclude by summarizing some of the things we have learned about the phase structure of QCD-like theories at finite temperature and chemical potential. We begin with the case of two massless flavors, figure 3 a. If we move along the chemical potential axis at temperature $T=0$, there is a minimum chemical potential required in order to introduce baryons into the system. Since nuclear matter is self-bound, this point is a first order transition: The density jumps from zero to nuclear matter density. Along the temperature axis, the line of first order transitions eventually ends in a critical point: This is the endpoint of the nuclear liquid-gas phase transition. If we continue to increase the chemical potential, we encounter the various phases of nuclear matter at high density. Many possibilities have been discussed in the literature, and we have nothing to add to this discussion. At even higher chemical potential, we encounter the transition to quark matter and the two flavor quark superconductor. Model calculations suggest that this transition is first order. This is also consistent with the fact that, asymptotically, the superconductor is of the first kind, and expected to exhibit a first order transition to the normal phase at the critical temperature for color superconductivity. In the case of two massless flavors, universality arguments suggest, and lattice calculations support, the idea that the finite temperature zero chemical potential chiral phase transition is second order. In this case, the line first order $\mu \neq 0$ transitions meets the $T \neq 0$ transition at a tricritical point 2232 .

This tricritical point is quite remarkable, because it remains a true critical point, even if the quark masses are not zero, figure $3 \mathrm{~b}$. A non-zero quark mass turns the second order $T \neq 0$ transition into a smooth crossover, but the first order $\mu \neq 0$ transition persists. While it is hard to predict where exactly the tricritical point is located in the phase diagram it may well be possible to settle the question experimentally. Heavy ion collisions at relativistic energies produce matter under the right conditions and experimental signatures of the tricritical point have been suggested 33 .

We have already discussed the phase structure of $N_{f}=3$ QCD with massless or light degenerate quarks in section 7 . We emphasized that at $T=0$ the low density, hadronic, phase and the high density, quark, phase might be continuously connected. On the other hand, there has to be a phase transition that separates the color-flavor locked phase from the $T=\mu=0$ hadronic phase. This is because of the presence of a gauge invariant $U(1)$ order parameter that distinguishes the two. In the case of $N_{f}=3$ massless flavors the finite temperature phase transition is known to be first order. We expect the transition from the superconducting to the normal phase at $T \neq 0$ and large $\mu$ to be first order, too. This means that there is no tricritical point in figure $3 \mathrm{c}$.

The phase diagram becomes more complicated if we take into account the effects of a finite strange quark mass, figure $3 \mathrm{~d}$. Consider increasing the strange quark mass in the color-flavor locked.phase. This shifts the Fermi momentum of the strange quarks with respect to the light quarks. If $m_{s}^{2} /(4 \mu)$ is larger than $\Delta\left(m_{s}=0\right)$ pairing between strange quarks and light quarks can no longer take place, and there is a first order transition to a phase (2SC) with separate pairing in the $u d$ and $s$ sectors

mbx: submitted to World Scientific on June 25, 2018 

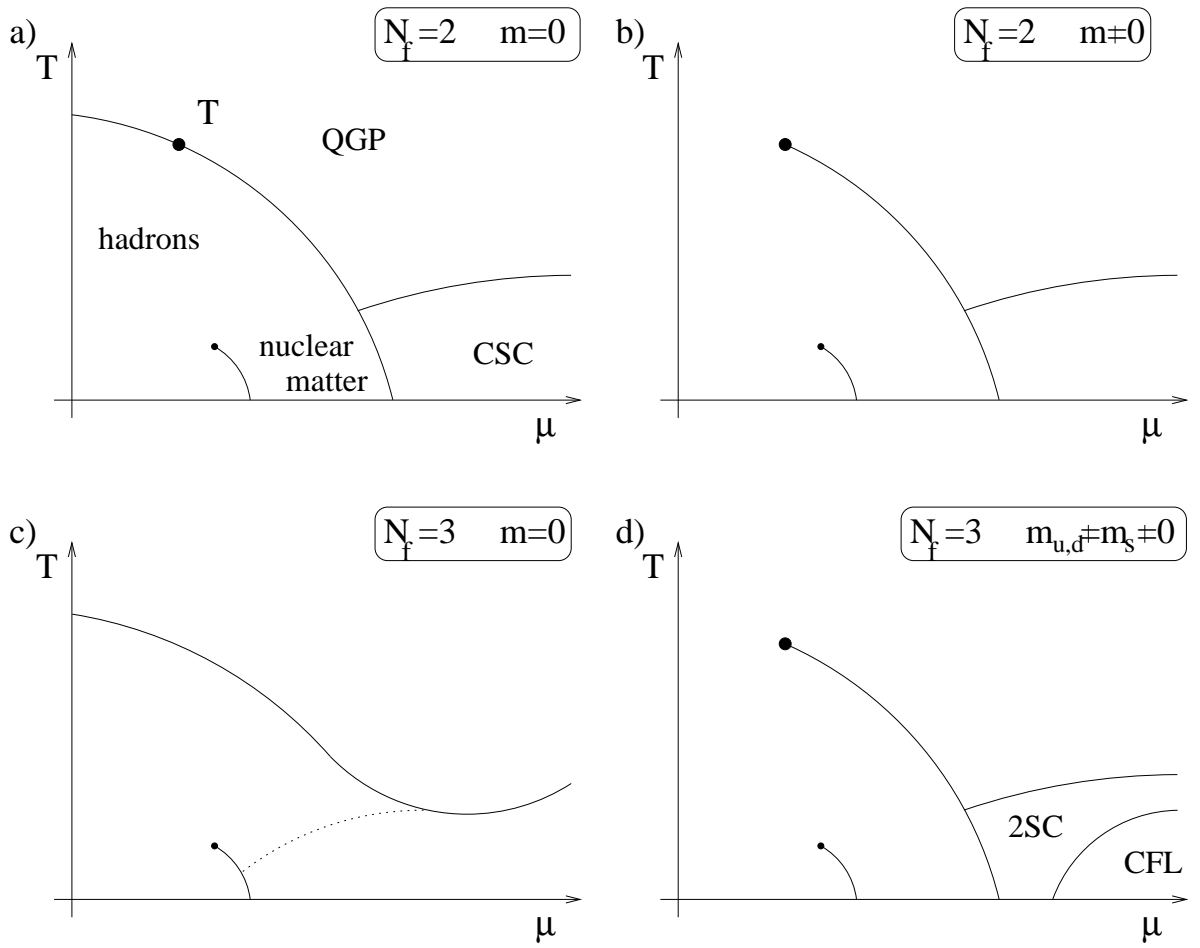

Figure 3. Schematic phase diagram of QCD at finite temperature and density. The figures a)-d) correspond to different numbers of massless and massive flavors, see the discussion in the text.

15126. Asymptotically the gap grows with $\mu$ and we expect the color-flavor locked phase to dominate for any value of $m_{s}$. Increasing the temperature or reducing the chemical potential favors the $2 \mathrm{SC}$ phase. Whether in the case of the physical value of the strange quark mass there is an interlude of the the 2SC phase along the $\mu \neq 0$ axis, instead of a direct transition between the CFL phase and nuclear matter, current calculations are not sufficiently accurate to decide. We know that there is at least one phase transition, because nuclear matter and the color-flavor locked phase are distinguished by a gauge invariant $U(1)_{s}$ order parameter. This, of course, is based on our belief that nuclear matter is stable with respect to strange quark matter or hyperonic matter. Current calculations have also not conclusively answered the question whether the transition along the $T \neq 0$ axis is a smooth crossover, as indicated in figure $3 \mathrm{~d}$ and favored by some lattice calculations, or whether the transition is first order, as would be the case if $m_{s}$ is sufficiently small. This is clearly an important question in connection with the existence of the tricritical point.

Thinking about QCD at finite baryon density has taught us many interesting lessons about the phase diagram, but there remain many question marks in the picture presented here, and much work remains to be done.

mbx: submitted to World Scientific on June 25, 2018 


\section{References}

1. S. C. Frautschi, in: Proceedings of the Workshop on Hadronic Matter at Extreme Energy Density, N. Cabibbo, Editor, Erice, Italy (1978).

2. F. Barrois, Nucl. Phys. B129, 390 (1977).

3. D. Bailin and A. Love, Phys. Rept. 107, 325 (1984).

4. S. Elitzur, Phys. Rev. D12, 3978 (1975).

5. E. Fradkin and S. Shenker, Phys. Rev. D19, 3682 (1979).

6. J. Polchinski, hep-th/9210046.

7. R. Shankar, Rev. Mod. Phys. 66, 129 (1995).

8. T. Schäfer and F. Wilczek, Phys. Lett. B450, 325 (1999).

9. N. Evans, S. Hsu, and M. Schwetz, Nucl. Phys. B551, 275 (1999); Phys. Lett. B449, 281 (1999).

10. D. T. Son, Phys. Rev. D59, 094019 (1999).

11. S. Hsu and M. Schwetz, preprint, hep-ph/9908314.

12. S. Weinberg, Nucl. Phys. B413, 567 (1994).

13. R. Rapp, T. Schäfer, E. V. Shuryak, and M. Velkovsky, Phys. Rev. Lett. 81, 53 (1998).

14. J. B. Kogut, M. A. Stephanov, D. Toublan, preprint, hep-ph/9906346.

15. T. Schäfer and F. Wilczek, Phys. Rev. D60, 074014 (1999).

16. M. Alford, J. Berges, and K. Rajagopal, preprint, hep-ph/9903502.

17. M. Alford, K. Rajagopal, and F. Wilczek, Nucl. Phys. B537, 443 (1999).

18. T. Schäfer and F. Wilczek, Phys. Rev. Lett. 82, 3956 (1999).

19. T. Schäfer, preprint, hep-ph/9909574.

20. M. Alford, K. Rajagopal, and F. Wilczek, Phys. Lett. B422, 247 (1998).

21. T. Schäfer and E. V. Shuryak, Rev. Mod. Phys. 70, 323 (1998).

22. J. Berges and K. Rajagopal, Nucl. Phys. B538, 215 (1999).

23. G. Carter and D. I. Diakonov, Phys. Rev, D60, 016004 (1999).

24. R. Rapp, T. Schäfer, E. V. Shuryak, and M. Velkovsky, preprint, hepph/9904353, to appear in Ann. Phys.

25. T. Schäfer and F. Wilczek, preprint, hep-ph/9906512.

26. R. D. Pisarski, D. H. Rischke, preprint, hucl-th/9907041.

27. D. K. Hong, V. A. Miransky, I. A. Shovkovy, and L. C. R. Wiejewardhana, preprint, hep-ph/9906478.

28. W. E. Brown, J. T. Liu, and H. Ren, preprint, hep-ph/9908310.

29. D. V. Deryagin, D. Yu. Grigoriev, abd V. A. Rubakov, Int. J. Mod. Phys. A7, 659 (1992).

30. E. Shuster and D. T. Son, preprint, hep-ph/9905448.

31. B.-Y. Park, M. Rho, A. Wirzba, and I. Zahed, preprint, hep-ph/9910347.

32. M.A. Halasz, A.D. Jackson, R.E. Shrock, M.A. Stephanov and J.J.M. Verbaarschot, Phys. Rev. D58, 096007 (1998).

33. M. Stephanov, K. Rajagopal, and E. Shuryak, Phys. Rev. Lett. 81, 4816 (1998). 